\title{
In Retrospect of Cloud Security Issues
}

\author{
Jvs. Arundathi, K.V.V.Satyanarayana
}

\begin{abstract}
Popular computing technologies like Distributed ,Parallel ,Grid etc., have already reached their peaks in providing services and now a hybrid aspect is capturing the focus which is a combination of traditional computing technology and network technology and termed to be "Cloud Computing ".A desperate demand for data sharing and handling enterprise applications have called upon for cloud computing .A blocking wind for leveraging cloud computing technology is the aspect of security .But the passion towards adopting cloud have overridden the security threats. This paper glances over various security threats, risks, challenges along with their resistance capabilities to overcome the vulnerabilities in the cloud and also some of the encryption techniques that are used in the cloud.
\end{abstract}

\section{Keywords: Encryption, Threats, Attacks ,cloud computing}

\section{INTRODUCTION}

According to NIST "Cloud computing is a model for enabling ubiquitous, convenient, on-demand network access to a shared pool of configurable computing resources (e.g., networks, servers, storage, applications, and services) that can be rapidly provisioned and released with minimal management effort or service provider interaction". In addition it has some other advantages like contributing low cost infrastructure ,flexibility ,scalability, collaboration and ease of use and also on-demand access from anywhere through the internet is being used by commercial entities and also by conventional users.

\section{Characteristics of cloud computing}

As stated in NIST definition, the cloud computing services have some aspects : Broad Network Access ,OnDemand Self Service, Rapid elasticity, Measure Service Resource Pooling[1][15] As per NIST cloud computing is described using four Deployment models and three service models

Deployment Models :There are four Deployment models in cloud[11][13].

A. Private Cloud is used with in the organization and its services and data cannot be accessed from the outsiders of the organization

B. Public Cloud has mega scalable infrastructure. It is retained and organized by academic, government or business organizations which provides services of the cloud open for the use of public.

C. Hybrid cloud is a combination of both private and public cloud usually private for sensitive data and strategic applications

Revised Manuscript Received on December 30, 2019.

* Correspondence Author

Jvs. Arundathi*, Phd Scholar, Department of Computer Science and Engineering, Kl Deemed to be University, Guntur, India.

Dr. K. V. V. Satyanarayana, Professor, M. Tech Coordinator, Department of Computer Science and Engineering, Kl Deemed to be University, Guntur, India.

(c) The Authors. Published by Blue Eyes Intelligence Engineering and Sciences Publication (BEIESP). This is an open access article under the CC BY-NC-ND license (http://creativecommons.org/licenses/by-nc-nd/4.0/)
D. Community cloud has a framework and services that can be provisioned for the use of specific community of the customers.

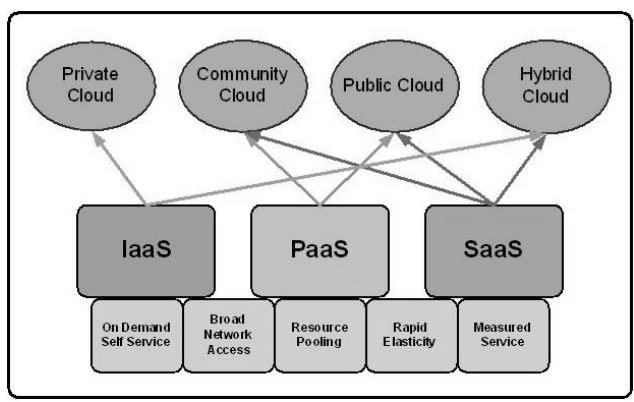

Figure 1: Cloud deployment model

\section{Cloud service models}

There are three types of service models in he cloud environment. User can select any one of the three services[10][13] based on their need ,They are:

A. SaaS(Software as Service): It is giving the ability to remotely use the software and its services on demand through the internet .It purges the huge responsibility of organizations such as setup, handling the installations, maintenance, and daily preservations. Ex: Face book, Whatsapp, Gmail etc.

B. PaaS (Platform as Service): it can be described as application development environments offered by the cloud provider as a service. It is the ability to deploy the user application on to the clouds infrastructure of provider. The development execution environment should be programming language, operating system and database. Example: Google App Engine.

C. IaaS(Infrastructure as Service):It provides the infrastructure such as hardware, servers, router ,storage and other modules of the networking to the users.

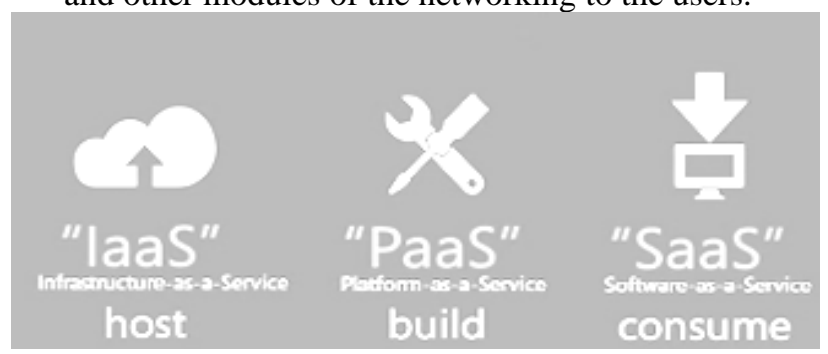

Figure 2 : Cloud Service models

The remaining paper is organised into sections. Section II discusses The cloud architecture, section III surveys various security open issues and threats,

section IV reviews security techniques, section V describes existing security algorithms . Finally section VI concludes the paper and future research scope. 


\section{In Retrospect of Cloud Security Issues}

\section{CLOUD ARCHITECTURE}

Cloud computing is a pool of resources which can be availed on demand based. It is available over the internet in a self service model without the service provider interaction

Cloud provides various products and services with technical , innovative opportunities. As per NIST's cloud computing reference architecture[2] there are five important actors[2] that can be influenced by cloud computing along with its security implications.

A. Cloud Consumer - An organization or a person that maintains a business relationship with , uses services from cloud providers

B. Cloud Provider - An organization or a person or entity responsible for making a service available to interested parties.

C. Cloud auditor- A party that can conduct independent assessment of cloud services. information system operations , security and performance of cloud implementation

D. Cloud Broker - An entity that manages the use, delivery and performance of cloud services and negotiates the relationship between consumers and providers of the cloud.

E. Cloud Carrier- An intermediary that provides transport and connectivity of cloud services from the cloud providers to cloud consumers [2]

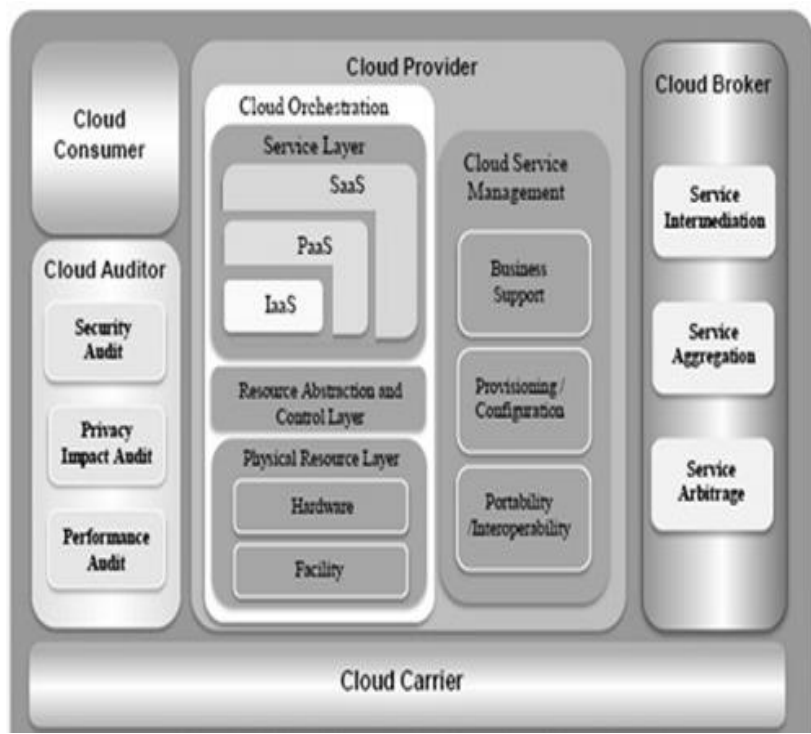

Figure 3: NIST reference cloud architecture[2]

\section{SECURITY OPEN ISSUES AND THREATS}

The adaption of cloud has been reached to the peak point and it is expected that more workloads will move to cloud from traditional local storage, ranging from the internet users to commercial organizations. While there are many security problems to be identified and analysed in various aspects like 1) Privileged User Access Management 2) Regulatory Compliance 3) Data Location 4) Data Segregation 6) Data protection and recovery support 7) Long Term Viability 8) Investigative support.

Cloud computing provides many benefits on other side it suffers from security issues which cannot be ignored. In the recent report of ENISA , thirteen technical risks were identified security challenges which are resulting from the cloud's
As per NIST's report cloud computing is facing some

wide range of outsourcing, Network Dependency, multi tenancy ,and scalability.

Fernandez et.al.[3] [6]provided complete retrospect of the research literature to define security challenges and open issues of cloud.

Main security challenges are

- Shared technologies vulnerabilities

- Data breach

- Account or service traffic hijacking

- Denial of service (DOS)

- Malicious insiders

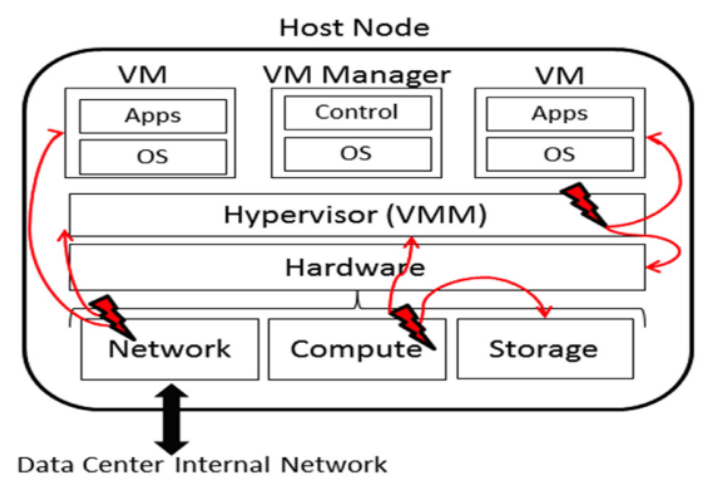

Figure 4: cloud platform attack vectors[6]

The above mentioned open issues can be generated by three main vectors of attack : Hypervisor ,Computing Hardware and Network[12] and the various attackers are internal users, External users and cloud provider (malicious employee)

Network is one of the most important vector in cloud platform with which the application can run

Hypervisor: It is a program that can enable you to host various distinct Virtual machines on a single hardware. The Hypervisor is also known as Virtual Machine Monitor(VMM).The Hypervisor presents the guest operating Systems with a virtual operating platform and it handles the execution of the guest operating systems. Hypervisor is the fundamental part that guarantee the multi tenancy feature in the cloud computing, The memory bus, disk bus, data and instruction caches and other VM instances are some of the physical recourses.[6]

External users can attack against the cloud infrastructure through the network. They can effect data confidentiality , integrity by tampering the communication channels. They can effect the availability of cloud provider data centres.

Internal Users (owners of VM instance) can exploit the hypervisor to attack another VM instance which is by the multi tenancy feature ie both the invader and the victim share the same host, which can breach the confidentiality of sensitive information[6]

The Cloud provider[8] itself might be an attacker. The employees could exploit their privileged position to steal the sensitive user information either by logical or physical manipulation of hardware platform .

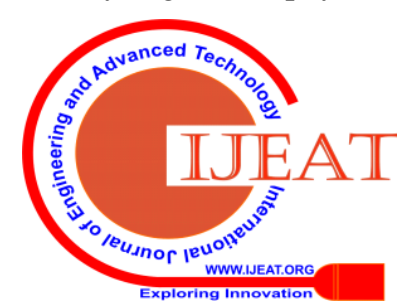


Table 1: [4] Cloud threats and attacks

\begin{tabular}{|c|c|c|c|}
\hline & TYPES & EFFECTS & SOLUTIONS \\
\hline \multirow{9}{*}{ Threats } & $\begin{array}{l}\text { Distinct service } \\
\text { receiving/delivery model }\end{array}$ & Control loss of cloud infrastructure & $\begin{array}{l}\text { Contributing the monitoring and } \\
\text { controlled services }\end{array}$ \\
\hline & Misuse of cloud computing & $\begin{array}{l}\text { Loss of validation, attack due to } \\
\text { unidentified sign-up ,deceit service }\end{array}$ & $\begin{array}{l}\text { Provide secured registration and } \\
\text { verification mechanisms by observing the } \\
\text { status of the network }\end{array}$ \\
\hline & Unsure API and interface & $\begin{array}{l}\text { Imprecise validation and verification, } \\
\text { data breach }\end{array}$ & $\begin{array}{l}\text { Use encryption during data transmission, } \\
\text { robust access control and verification } \\
\text { methods }\end{array}$ \\
\hline & Harmful insiders & $\begin{array}{l}\text { Permeating resources, demolishing the } \\
\text { assets, productivity loss, etc.. }\end{array}$ & $\begin{array}{l}\text { Notify the breaches, report the agreement } \\
\text {,use the transparent security management } \\
\text { technique }\end{array}$ \\
\hline & $\begin{array}{l}\text { Issues due to Shared } \\
\text { technology }\end{array}$ & $\begin{array}{l}\text { Obstruction of user services by } \\
\text { negotiating hypervisor }\end{array}$ & $\begin{array}{l}\text { For administrative task use strong } \\
\text { verification and access control methods, } \\
\text { Audit vulnerability and configuration }\end{array}$ \\
\hline & Leakage and Loss of Data & $\begin{array}{l}\text { Destruction, corruption, modification } \\
\text { or deletion of sensitive data }\end{array}$ & $\begin{array}{l}\text { Provide data backup and storage } \\
\text { techniques }\end{array}$ \\
\hline & $\begin{array}{l}\text { Hijacking of Account } \\
\text { /Service }\end{array}$ & $\begin{array}{l}\text { Hijacking user credentials, cloud } \\
\text { critical area access, conceding the } \\
\text { service security to the attacker }\end{array}$ & $\begin{array}{l}\text { Following the substantial verification } \\
\text { mechanisms, secured policies, and secure } \\
\text { link. }\end{array}$ \\
\hline & Delineation of Risk & $\begin{array}{l}\text { Policies, operations related to internal } \\
\text { security, etc.. }\end{array}$ & $\begin{array}{l}\text { Using monitoring and altering system to } \\
\text { secure the data }\end{array}$ \\
\hline & Piracy of Identity & $\begin{array}{l}\text { An intruder can get identity of the } \\
\text { valid user and misuse that. }\end{array}$ & $\begin{array}{l}\text { Using robust multi-tier passwords and } \\
\text { verification methods }\end{array}$ \\
\hline \multirow{9}{*}{ Attacks } & Zombie attack(DoS/DDos ) & $\begin{array}{l}\text { The availability of service may be } \\
\text { affected }\end{array}$ & Robust verification and validation \\
\hline & Service Injection & $\begin{array}{l}\text { The integrity of the Service will be } \\
\text { distressed, instead of valid service } \\
\text { malicious service will be provided }\end{array}$ & $\begin{array}{l}\text { Robust isolation mechanisms between } \\
\text { VMs, To check service integrity use hash } \\
\text { function }\end{array}$ \\
\hline & $\begin{array}{l}\text { Attack on Hypervisor } \\
\text { /Virtualization }\end{array}$ & $\begin{array}{l}\text { Access the user credentials and } \\
\text { control }\end{array}$ & $\begin{array}{l}\text { Need security solutions of a hypervisor, } \\
\text { monitor its activities, VM isolation needed }\end{array}$ \\
\hline & Attacks from user to root & $\begin{array}{l}\text { Affect the privacy of user's } \\
\text { sensitive information and services }\end{array}$ & $\begin{array}{l}\text { Use robust password, better verification } \\
\text { mechanisms }\end{array}$ \\
\hline & Port Scanning & $\begin{array}{l}\text { Abnormal behaviour of the service, } \\
\text { service availability is affected }\end{array}$ & Required robust port security \\
\hline & Man_in_the middle & $\begin{array}{l}\text { The security and privacy of the data } \\
\text { is invaded }\end{array}$ & $\begin{array}{l}\text { A proper (SSL)secure Secure Socket Layer } \\
\text { architecture is required }\end{array}$ \\
\hline & Metadata Spoofing & $\begin{array}{l}\text { Abnormal behaviour of the service, } \\
\text { privacy of the service may be affected }\end{array}$ & $\begin{array}{l}\text { The functionality of the Service and other } \\
\text { details should be encrypted, robust } \\
\text { verification mechanism is needed to access } \\
\text { the required file }\end{array}$ \\
\hline & Phishing & $\begin{array}{l}\text { The privacy of the user credentials } \\
\text { will be affected }\end{array}$ & Use HTTPS \\
\hline & Backdoor Channel Attack & $\begin{array}{l}\text { Affects the availability of service, } \\
\text { data privacy }\end{array}$ & $\begin{array}{l}\text { robust verification, and isolation } \\
\text { mechanisms are needed }\end{array}$ \\
\hline
\end{tabular}

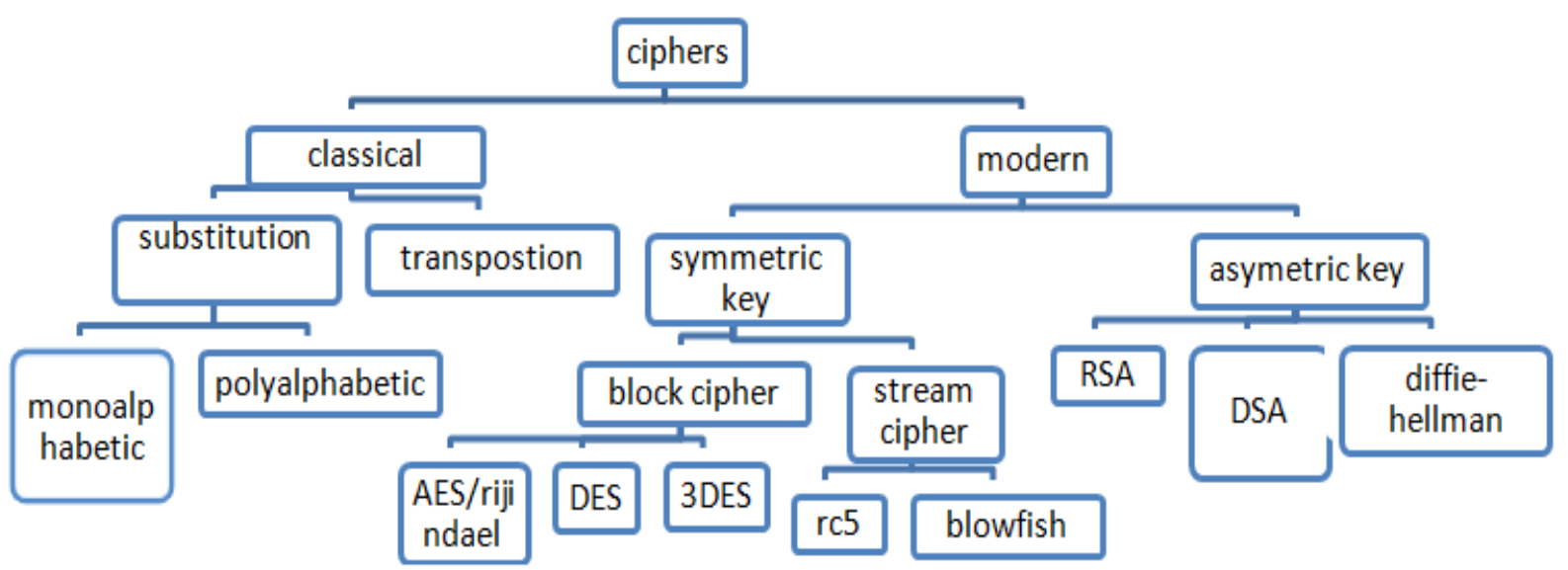

Figure 5: classification of encryption methods 
In Retrospect of Cloud Security Issues

Table 2: Various security issues of cloud computing

\begin{tabular}{|c|c|c|}
\hline & & Data storage issue \\
\hline & & Un-trusted computing \\
\hline & Security issues related to data storage and & Availability of Data and service \\
\hline & computing & Cryptography \\
\hline & & Recycling of Cloud data \\
\hline & & Malware \\
\hline & & Image Management of VM \\
\hline & & Virtual Machine Monitor \\
\hline & Security issues related to Virtualization & Virtualization of Network \\
\hline & & mobility \\
\hline & & Virtual Machine Issues \\
\hline & & Malware \\
\hline & & $\begin{array}{l}\text { Cutting edge threats } \\
\text { and vicious outsider }\end{array}$ \\
\hline & Security Issues related to Internet and & Internetworking Protocols \\
\hline & Services & Web Services \\
\hline & & Web Technologies \\
\hline & & Availability of Service \\
\hline Security & Network Security Issues & Mobile platforms \\
\hline Issues of & & Circumference security \\
\hline & & Physical access \\
\hline & & User credentials \\
\hline & & Entity authentication \\
\hline & Access control issues & Authorization \\
\hline & & Management of user identity \\
\hline & & Anonymization \\
\hline & Software security issues & Platform and frameworks \\
\hline & & User frontend \\
\hline & & Cloud to cloud trust \\
\hline & Trust management issues & Human aspect \\
\hline & & Reputation \\
\hline & & Dependency on the audit reports \\
\hline & & Anonymization \\
\hline & & Forensics \\
\hline & & Acts \\
\hline & Aspects like legal and compliance & Legal problems \\
\hline & & Incorrect resource usage metering \\
\hline & & Governance \\
\hline
\end{tabular}

Table 3:Security challenges and risks

\begin{tabular}{|l|l|}
\hline Threats & Description of Risk \\
\hline DoS & $\begin{array}{l}\text { In this attack, the invader will perform flooding on the server to make the services and/or resources to be } \\
\text { unavailable to the users of the cloud. }\end{array}$ \\
\hline DDoS & $\begin{array}{l}\text { A Distributed DoS attack is an attempt to generate services that are unavailable by devastating it with traffic } \\
\text { from various machines that are scattered over theInternet. }\end{array}$ \\
\hline MitM & $\begin{array}{l}\text { A Man-in-the-Middle attack is a type of eavesdropping attack where an invader involves himself in a } \\
\text { communication between two parties, steals important information from the users, and then passes to the third } \\
\text { party. }\end{array}$ \\
\hline IP Spoofing & $\begin{array}{l}\text { IP Spoofing is a process to gain access to the server in unauthorized manner thereby an intruder unjustifiably } \\
\text { impersonates the IP address of trusted host to conceal his identity. }\end{array}$ \\
\hline Packet Sniffing & $\begin{array}{l}\text { The analyzer or Packet Sniffer is used to determine the problems related to network.. However, an intruder } \\
\text { can capture and analyze all transmitted sensitive information and also he can use it. }\end{array}$ \\
\hline Port Scanning & Attacker sends queries to search for vulnerable ports on the server and attempts to identify kind of used service. \\
\hline Session Hijacking & An attacker can hijack an active session and masquerade as one of the conversation parties. \\
\hline Phishing & Phishing is the attempt to steal sensitive user data such as credit card details and passwords, usernames. \\
\hline
\end{tabular}

\section{SECUTIRY TECHNIQUES}

In the present era various number of applications are relying on internet such internet banking ,online shopping , digital bill payment, stock trading etc., All these public networks need to have the end to end connection in a secured manner which should also be confidential in order to ensure data authentication, confidentiality ,availability integrity as well as accountability.

"As per NIST computer security can be defined as the protection afforded to an automated information system in order to attain the applicable objectives of preserving the integrity ,availability of confidentiality of information system resources"(inclusion firmware, hardware, software, data /information and tele-communications)

Security is the process of protecting the services and information from an unauthorised access, modification or destruction. In networking the security can be obtained by using cryptography (one of science and art) is of transforming the messages so that they can be protected by attacks.

Published By:

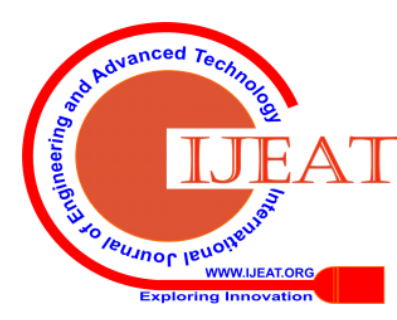


Encryption: is one of important mechanism which can ensure the security of sensitive information. The encryption algorithms are classified into two groups: asymmetric key(public key) and symmetric key(secret key) and [5][7][9] Symmetric key: is also known as conventional encryption, is a form of cryptosystem in which encryption and decryption performed using the same key[5][7][9]

Asymmetric encryption : In which encryption and decryption are performed using different keys ie., public key, private key[5][ 9].

\section{EXISTING ALGORITHMS FOR SECURITY}

In data communication encryption plays a major role to secure the data. The encryption algorithms[7][14] used in cloud computing are

\section{A. Symmetric encryption algorithms:}

In this method sender and receiver use a single secret key which is used to encrypt and decrypt the messages. Some of the encryption algorithms are

a) DES(Data Encryption Standard): It is a symmetric key algorithm used to encrypt the information. It was developed by IBM in 1975. In DES algorithm block cipher is of 64bits and key is of 56bits. Now a days this

b) 3DES(Triple Data Encryption algorithm): It is developed to overcome the flaws of DES without designing a new crypto system. It was developed by IBM in 1978. 3DES extends the key size of DES by applying the algorithm 3 times in succession with 3 different keys. The combined key size is thus 168 bits( 3 times to DES( $3 * 56)$ ).TDEA uses three 64bit keys $\mathrm{K} 1, \mathrm{~K} 2, \mathrm{~K} 3$ in Encrypt-Decrypt-Encrypt(EDE) mode. 3DES is slower than other block cipher methods.[5][14]

c) AES(Advanced Encryption Standard): Is one of the new encryption standard recommended by NIST to replace DES in 2001. The AES can support any combination of data (128bits ) and key length of 128,192 and 256 bits. During encryption and decryption process AES goes through 10 rounds for 128 bit keys, 12 rounds for 192 bit keys and 14 rounds for 256 bit keys to deliver final cipher text. The draw back of this algorithm is difficult to implement.[5][7]

d) Blowfish: It is a symmetric key algorithm designed by Bruce Schneier in 1993. It is a basic algorithm developed an option to DES algorithm to overcome many problems that come with many other algorithms. This algorithm is available in public domain. And can be available to free to every one.[7].The key size is 32448 bits and 16 rounds.

e) RC5(Rivest Cipher): It is a symmetric key algorithm. Mainly known for its simple execution. This is developed by Ronald Rivest in the year 1994. The speed of algorithm is slow comparative to remaining algorithms

\section{B.Asymmetric Algorithms:}

These are public key algorithms which generally make use of different keys for encryption algorithm is insecure for lots of applications.[5][14]

and decryption. These algorithms are most important because these can be used for transmission of encryption keys.

a) RSA (Rivest-Shamir-Adeleman): Is the most simple and common asymmetric algorithm used for both encryption and decryption of digital signature. It has fast encryption key[5][14. It was developed by Ronrivest,Adi shamir,and Leonard Adleman in 1978.

b) DSA : It is an important algorithm for processing the digital data. It was given by the NIST in 1991.

c) Diffie-Hellman : It is the earlier asymmetric data standard algorithm developed in the year 1976. This algorithm most widely used key exchange algorithm[14]

\section{RESULT ANALYSIS}

Table 4: comparison of performance of various algorithms[19].

\begin{tabular}{|c|c|c|c|c|c|}
\hline SNO & Algorithm & $\begin{array}{c}\text { Key } \\
\text { size(bit) }\end{array}$ & $\begin{array}{c}\text { File } \\
\text { size(bytes) }\end{array}$ & $\begin{array}{c}\text { Avg } \\
\text { Encryption } \\
\text { time(msec) }\end{array}$ & $\begin{array}{c}\text { Avg } \\
\text { Decryption } \\
\text { time(msec) }\end{array}$ \\
\hline \multirow{3}{*}{1} & \multirow{3}{*}{ AES } & \multirow{3}{*}{256} & 329 & 287 & 293 \\
\hline & & & 778 & 299 & 304 \\
\hline & & & 2048 & 300 & 297 \\
\hline \multirow{3}{*}{2} & \multirow{3}{*}{ BLOWFISH } & \multirow{3}{*}{128} & 329 & 293 & 290 \\
\hline & & & 778 & 287 & 278 \\
\hline & & & 2048 & 283 & 279 \\
\hline \multirow{3}{*}{3} & \multirow{3}{*}{ DES } & \multirow{3}{*}{56} & 329 & 284 & 280 \\
\hline & & & 778 & 292 & 282 \\
\hline & & & 2048 & 303 & 317 \\
\hline \multirow{3}{*}{4} & \multirow{3}{*}{ RC4 } & \multirow{3}{*}{64} & 329 & 282 & 286 \\
\hline & & & 778 & 283 & 280 \\
\hline & & & 2048 & 313 & 292 \\
\hline \multirow{3}{*}{5} & \multirow{3}{*}{ RSA } & \multirow{3}{*}{1024} & 329 & 462 & 499 \\
\hline & & & 778 & 541 & 450 \\
\hline & & & 2048 & 488 & 491 \\
\hline
\end{tabular}

The experimental result for some of the encryption algorithms like AES,Blowfish,DES,RC4 and RSA have been implemented on several file sizes:329,778 and 2048 bytes respectively. The encryption and Decryption time are calculated in milliseconds and input size is taken in kilo bytes

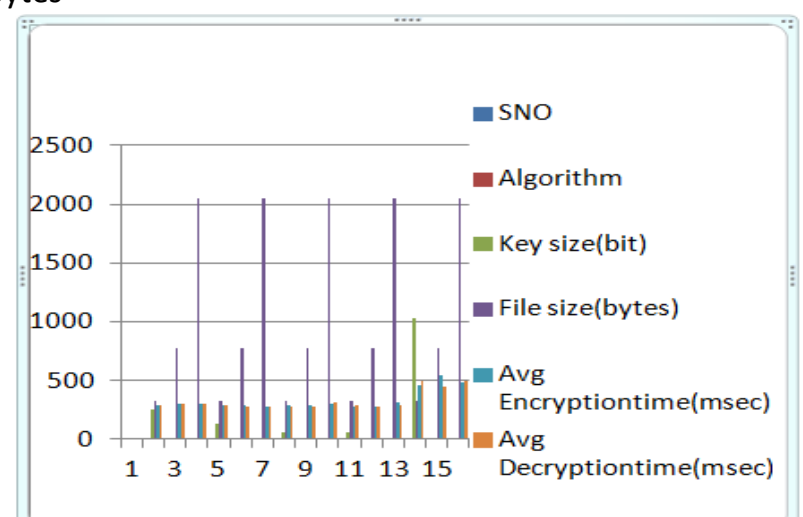

Figure6: Encryption time of different algorithms[19].

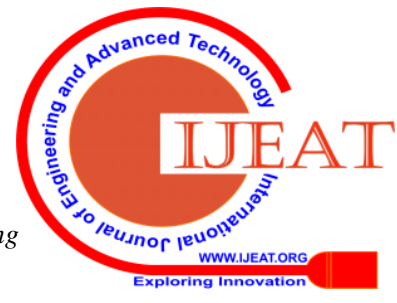




\section{In Retrospect of Cloud Security Issues}

From the above figure we can conclude that the asymmetric encryption/decryption techniques are slower than the symmetric encryption /decryption techniques. All the algorithms except DES and RSA algorithms there is a proportion relation between input file size running time. As the input file size increases the running time of DES and RSA changes slightly. Compare to all the algorithms RSA will take more time for both encryption and Decryption.

\section{CONCLUSION}

Cloud computing provides a various assets of cloud services and resources in various fields. However Cloud security issues linger the extensive obstacles that may hinder the massive adoption of cloud computing. Security engineering is one of the best practices to provide the best mechanisms and approaches for establishing services and systems for sustainability, security and resiliency. In this paper the overview of various security threats, attacks with solutions, security issues, challenges, risks and some of the encryption techniques were also discussed, which are facing some problems regarding speed and key length. It can be solved by using some hybrid techniques. As cloud services have huge number of domains, deployment models and respective algorithmic approaches since there is a problem in cloud security to avail the services (storage, infrastructure etc.)there is a huge scope for research.

\section{REFERENCES:}

1. K.H.A-Ai-shqueerat,et al.,"Cloud Computing Security Challenges in Higher Educational Institutional -A Survey” IJCA - volume 161,No 6, march 2017 page no22- 29.

2. Gururaj Ramachandra et al., "A Comprehensive Survey on Security in Cloud Computing”, Elesevier - procedia Computer Science(2017) pp no : 465-472.

3. Fernandes D.A. etal., "Security Issues in Cloud Environment a Survey" April 2014:13(2):113-70.

4. Ashish Singh, Kakali Chatterjee,et al., "Cloud Security Issues and Challenges: A Survey" Journal of Network and Computer Applications2015.

5. Supriya,GurpreetSingh "A Study of Encryption Algorithms (RSA, DES, 3DES and AES) for Information Security", International Journal of Computer Applications (0975 - 8887) Volume 67- No.19, April 2013.

6. Luigi coppolino,Salvator D'Antonio,Giovanni Mazzeo,Luigi Ramano "Cloud security: Emerging threats and current solutions", Elseviercomputer and Electrical Engineering 000(2016) 1-15

7. Geetha V,Lavanya N,Priyadharshiny S, Sofeiyakalaimathy C" Survey on Security Mechanisms for Public Cloud Data",2016 IEEE.

8. Subhashis Sengupta,Vikrant Kaulgud,Vibhu Saujanya Sharma," Cloud computing Security-Trends and Research Directions" ,2011 IEEE.

9. Tanvi Agrawal,S K Singh,” Analysis of Security Algorithms in Cloud Computing",2016 IEEE.

10. Saurabh Singh,Young-sik Jeong,Jong Hyuk park," A Survey on Cloud Security :Issues, Threats and Solutions” ,Journal of Network and Computer Applications-Elsevier 2016.

11. Pankaj Singhai, et.al" ASurvey on Cloud Security Issues and Challenges",IJIRCCE,Vol.5,Issueue 5,May 2017.

12. Umar Mukhtar Ismail,Syed Islam "Towards Cloud Security Monitoring :A Case Study"2016 Cyber and Cyberforensics Conference,2016 IEEE.

13. Mohammad Ubaidullah Bokhari,Qahtan Makkishallal"Security and Privacy issues in cloud computing",2016 IEEE.

14. Er Ashima Pansotra et.al "Cloud SecurityAlgorithms",Inetrnational Journal of Security and its Applications,vol.9,No.10(2015) pp.353360 .
15. R Sharma and R K Trivedi-Literature Review: Cloud [16]. Syed,Heena I and Naghma A Baig "Survey on Cloud Computing"IJETAE,2013.

16. Mazhar Ali,SAmee U.Khan,Athanasios V,Vasilakos,"Security in Cloud Computing:Opportunities and Challenges"Accepted Manuscript,Elsevier,2015.

17. R.Barona,E.A.Mary Anita,"A Survey on Data Breach Challenges in Cloud Computing Security: Issues and Treats",2017,ICCPCT,IEEE

18. Prachi Garg,et al,"Security Techniques for Cloud Computing Environment,ICCCA2017,IEEE.

19. Md.Alam Hossain, et al.," Performance Analysis of Different Cryptography Algorithms” ,IJARCSSE,Vol2,206

\section{AUTHORS PROFILE}

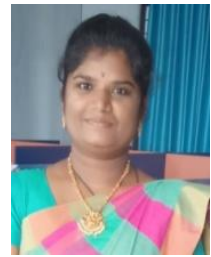

Ms. J V S ARUNDATHI, is currently a $\mathrm{PhD}$ scholar in the Department of Computer Science and Engineering, KL Deemed To Be UNIVERSITY, GUNTUR, INDIA. Her research interest includes Information Security and Cloud Computing Security, IOT.

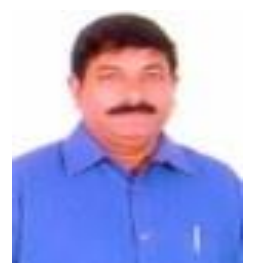

Dr. K.V.V. SATYANARAYANA, is a Professor and M. TECH Coordinator in the Department of Computer Science and Engineering, KL Deemed To Be UNIVERSITY, GUNTUR, INDIA. His research interest includes Information Security and Cloud Computing Security. He is the Senior Life Member of Computer Society. 